\section{Caciar mitilin}

Oct. 3-5. Advances in Recombinant DNA Technology: Genetic Engineering and the Immune System. Incline Village, NV. Info: Symposium Coordinator, The Center for Advanced Training in Cell and Molecular Biology, The Catholic University of America, Washington, DC 20064

Oct. 4-7. Biotek India '88. New Delhi. Info: Convex Division of Applied Technology Services, 14F, Basant Lok, VasantVihar, New Delhi - 110 057, India.

Oct. 5-7. The PaineWebber-Bio/Technology Conference: Biopharmaceuticals Futures. San Diego, CA. Info: Diana Berger, Biopharmaceuticals Futures, Nature Publishing Co., 65 Bleecker St, New York, NY 10012-2467 (Phone: 212-4779600).

Oct. 11-13. The British Laboratory Week. Grand Hall, Olympia, London. Info: Curtis Steadman \& Partners Limited, The Hub, Emson Close, Saffron Walden, Essex, CB10 1HL U.K

Oct. 11-15. American Society of Human Genetics 1988 Annual Meeting. New Orleans, LA. Info: Peggy Gardiner, Meetings Manager, American Society of Human Genetics, 9650 Rockville Pike, Bethesda, MD 20814

Oct. 20-21. NEBA Worcester Colloquium V. Worcester, PA. Info: Dr. Judith E. Miller, Dept. of Biology and Biotechnology, Worcester Polytechnic Institute, Worcester, MA 01609

Oct. 20-21. The Regulation of Medical Software. Rockville, MD. Info: Health Industry Manufacturers Association, Meetings Dept., 1030 Fifteenth St. N.W., Washington, D.C. 20005-1598.

Oct. 23-26. 28th Annual Interscience Conference on Antimicrobial Agents and Chemotherapy. Los Angeles, CA. Info: Mr. Robert D. Watkins, Director, Public Affairs, American Society for Microbiology, 1913 I St., N.W., Washington, D.C. 20006.

Oct. 24-25. Frontiers of Chemistry: Biotechnology. Columbus, OH. Info: Dr. Stobaugh, Research Dept., Chemical Abstracts Service, P.O. Box 3012, Columbus, $\mathrm{OH} 43210$.

Oct. 25. National Institutes of Health/Alcohol, Drug Abuse, and Mental Health Administration: Industry Collaboration Forum. Washington, D.C. Info: Pat Davenport, Courtesy Associates, Inc., Suite 300,65515 th St., N.W., Washington, D.C. 20005 .

Oct. 26. New Business Opportunities in Peptides: A Free Executive Briefing. Secaucus, NI. Info: Lori Donofrio-Galley, Seminar Manager, Strategic Analysis Inc., Box 3485, R.D.3, Fairlane Rd., Reading, PA 19606.
Oct. 30-Nov. 5. Sixth International Congress of Culture Collections. College Park, MD. Info: Bobbie Brandon, American Type Culture Collection, 12301 Parklawn Dr., Rockville, MD 20852.

Oct. 31-Nov. 2. Growth Factors for Wound Healing. New Haven, CT. Info: Technology Management Group, 25 Science Park, New Haven, CT 06511.

Oct. 31-Nov. 2. Eighth International Symposium on HPLC of Proteins, Peptides and Polynucleotides. Copenhagen, Denmark. Info: Secretariat, Eighth ISPPP, c/o DIS Congress Service, Linde Allé 48, DK-2720 Vanløse, Copenhagen, Denmark.

Oct. 31-Nov. 3. The 1988 American Association of Pharmaceutical Scientists Annual Meeting. Orlando, FL. Info: AAPS, 60 King St., Alexandria, VA 22314-3105.

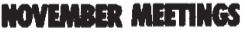

Nov. 2-4. Liquid Chromatography Mass Spectroscopy (LC/MS; SFC/MS; MS/MS). Freiburg i. Br., West Germany. Info Workshop Office IAEAC, M. FreiHäusler, Postfach 46, CH-4123 Allschwil 2, Switzerland.

Nov. 2-4. Viruses: New Leads into Cellular Processes. Piscataway, NJ. Info: Office of Continuing Education, The State University of New Jersey-Rutgers, 675 Hoes Lane, Piscataway, NJ 08854.

Nov. 3-4. DNA Probes in the Practice of Medicine. San Diego, CA. Info: American Medical Association, 535 North Deerborn St., Chicago, IL 60610 .

Nov. 3-4. National Conference on Biotechnology Ventures. Redwood City, CA. Info: Ken Lee, Arthur Young, 660 Hansen Way, Palo Alto, CA 94304

Nov, 4-6. Third Annual Conference on Clinical Immunology. San Francisco, C.A. Info: Clinical Immunology Society, P. O Box 827, Bowie, MD 20715.

Nov, 6-9, Biomodulation of Cancer Berkeley, CA. Info: Society for Biological Therapy 1988, c/o A.S. M. Meetings, 1913 Eye St. N. W., Washington, DC 20006

Nov, 6-9. Third Annual International Pharmaceutical Engineering Forum. Clearwater Beach, FL. Info: ISPE, 8910 N. Dale Mabry, Suite 27, Tampa, FL 33614.

Nov. 7-8. European Conference on Biotechnology: Scientific, Technical and Industrial Challenges. Verona, Italy. Info: Marc Chopplet, European Institute of Technology, Biotechnology Program, Tour Franklin-Cedex 11, 92081 Paris La Defense, France
Nov. 14-16. Biotech USA: The 5th Annual Industry Conference and Exhibition. San Francisco, CA. Info: Biotech USA '88, c/o CMC, 200 Connecticut Ave., Norwalk, CT 06856-4990 Tel: 1-800-243. 3238 ext.232.

Nov, 16-19. Biotec '88. Düsseldorf, West Germany. Info: Düsseldorfer Messegesellschaft mbH, NOWEA, Kongreßbüro BIOTECH '88, Postfach 320203 , D-4000 Düsseldorf 30 , West Germany.

Nov. 21-22. Recent Progress in Cytokine Research. Tokyo, Japan. Info: Secretariat, Masahiro Nobuhara, Director, Life Science Affairs, Mochida Pharmaceutical Co., Ltd., 1-7, Yotsuya, Shinjuku-ku, Tokyo 160 , Japan.

Nov. 29-Dec. 1. Equipment Design for Bioprocess Engineering Colloquium. Chicago, IL. Info: See Nov. 28

Nov, 29-Dec. 1. BioChemE '88. Washington, DC. Info: American Institute of Chemical Engineers, 345 East 47th St. New York, NY 10017.

\section{JTante armes}

Invitron Corp. (St. Louis, MO) named Randall G. Rupp vice president for process development; Victor A. Jegede, Ph.D vice president of regulatory affairs and quality assurance, and Richard R. Paris vice president for plant operations.

Genentech (South San Francisco, CA named Frederick M. Hoar vice president, corporate communications, and Stephen Raines vice president, patents.

BioTechnica Agriculture (Overland Park KS), a subsidiary of BioTechnica International, Inc., named Virgil W. Smail vice president of corporate development.

Embrex (Research Triangle Park, NC) hired Kenneth P. West vice president for business development.

Pharmaceutical Manufacturers Association (Washington, DC) promoted Charles B. Cleveland to associate vice president for research and development.

Synergen (Boulder, CO) appointed Wil liam D. Fairbairn director of regulatory affairs.

Biotherapeutics (Franklin, TN) elected Martin Dale to its board of directors

Cygnus Research Corp. (Redwood City, CA) announced that Gordon L. Flynn, $\mathrm{Ph} . \mathrm{D}$. joined the company as vice president of basic research.

Max Planck Institute of Biochemistry (Munich, Germany) named Axel Ullrich as its new director. Mr. Ullrich had been a staff scientist at Genentech (South San Francisco, CA). 